\title{
Self-organization of hollow-cone carbonate crystals through molecular control with an acid organic polymer
}

\author{
Yuya Oaki, Ryota Adachi and Hiroaki Imai \\ Hollow-cone morphologies have been formed on carbonate crystals of calcite, aragonite and vaterite through molecular control \\ with an organic acid polymer. The macroscopic conical shapes featured hollow interiors and consisted of oriented nanocrystals \\ mixed with this organic polymer, or mesocrystals. At submicrometer scales, the mesocrystal structures are mediated by the \\ interaction of the organic polymers. The macroscopic hollow-cone shapes were formed through the directed growth of the \\ mesocrystals under diffusion-controlled conditions. Because biological macromolecules control the growth of biominerals, \\ a molecular-control approach was used in this study to induce the self-organization of hierarchical architectures. \\ Polymer Journal (2012) 44, 612-619; doi:10.1038/pj.2012.29; published online 21 March 2012
}

Keywords: acidic organic polymer; biomineralization; calcium carbonate; crystallization; fusion materials; molecular control; self-organization

\section{INTRODUCTION}

In biomineralization, functional organic/inorganic composites are formed through self-organized crystal growth. Biological macromolecules control the crystal growth, leading to the formation of hierarchical structures and complex morphologies in biominerals. ${ }^{1-3}$ We can learn synthetic processes, specifically, molecular-control approaches, from biomineralization. ${ }^{4-14}$ In the present study, the hollow-cone morphologies based on carbonate mesocrystals were prepared through a molecular-control approach with an organic polymer.

A variety of carbonate-based biominerals, such as nacreous layers, sea urchin spines and eggshell, have specific macroscopic morphologies. In previous works, we found that oriented nanocrystals mixed with organic macromolecules produce macroscopic shapes with single-crystalline orientation. ${ }^{15-17}$ These mesocrystal structures have attracted considerable interest in recent years. ${ }^{18-20}$ Learning from biomineralization, mesocrystal structures have been synthesized with synthetic organic molecules. Biomimetic morphological control of crystals has been studied in a variety of inorganic materials using organic molecules. Kato and co-workers have reported that the cooperative effects of organic macromolecules facilitate the formation of organic/inorganic composites. ${ }^{21-28}$ The goal of the present study is the development of rational molecular-control approaches for the direction of crystal growth, as observed in biomineralization.

Based on previous reports, ${ }^{18-33}$ organic molecules have three main roles in crystallization processes: templates for crystallization, additives for growth control through the interaction at crystal faces and agents for diffusion control. Organic macromolecules serve as templates for crystal growth through heterogeneous nucleation (Effect I). For example, calcium-carbonate thin films have been fabricated on various substrates. ${ }^{21-29}$ The molecular recognition between the substrates and the crystal faces initiates templated growth. The formation of mesocrystals, or oriented nanocrystals, is induced by controlled crystal growth in the presence of organic molecules (Effect II). ${ }^{17-20,30,31}$ The organic molecules and polymers can adsorb onto the crystal faces. Because the adsorption of organic molecules onto crystals induces the segmentation of crystal growth through periodical inhibition, mesocrystal structures are formed with the incorporation of organic molecules. The diffusion-controlled conditions required for crystal growth can be mediated by organic molecules, such as polymers (Effect III). ${ }^{26,27,32,33}$ The diffusion rate and direction affect the morphogenesis of crystals. The combination of these three effects facilitates the control of crystal morphologies through biomimetic routes. In the present work, the combination of Effects II and III leads to the formation of hollow-cone $\mathrm{CaCO}_{3}$ architectures consisting of the mesocrystals.

Hollowed morphologies consisting of carbonate crystals were observed in coccoliths, which are found in nature. Hollow-cone and hopper-shaped architectures have been synthesized in a number of inorganic materials. ${ }^{34-40}$ In earlier studies, hollow-cone crystals of barium sulfate were synthesized through polymer-mediated crystallization with double-hydrophilic copolymers. ${ }^{34,35}$ Hollow-cone 
vaterite crystals have been formed at the interface between solutions and air. ${ }^{36}$ The electrolytic deposition of $\mathrm{CaCO}_{3}$ has produced micrometer-sized cups of vaterite on silicon substrates. ${ }^{37}$ Trumpetshaped calcite crystals have been prepared in the presence of a chelating agent. ${ }^{38}$ Hopper-shaped lead telluride and copper sulfide have been synthesized through hydrothermal and solvothermal syntheses. ${ }^{39,40}$ However, the formation conditions and mechanisms of the hollow architectures have not been fully clarified in these previous works. In general, the morphologies of crystals are transformed from polyhedral shapes into hopper and skeletal morphologies with an increase in the influence of the diffusion processes required for crystallization. Therefore, it is inferred that the diffusion-controlled conditions for crystal growth involve the morphogenesis of hollow-cone architectures.

In this study, we have synthesized hollowed carbonate crystals with vaterite and calcite, which are polymorphs of $\mathrm{CaCO}_{3}$, and aragonite, a polymorph of barium carbonate $\left(\mathrm{BaCO}_{3}\right)$, in the presence of poly(acrylic acid) (PAA). Mesocrystal structures consisting of oriented nanocrystals measuring $<100 \mathrm{~nm}$ were observed in the hollow-cone morphologies. The synthetic organic polymer had an essential role in the morphogenesis of the hollow-cone architectures. The interaction between PAA and the specific crystal faces led to the formation of the mesocrystals. The generation of macroscopic morphologies was achieved under diffusion-controlled conditions. The molecular-control approach used to form the hierarchical crystal morphologies can be applied to a wide variety of other materials.

\section{EXPERIMENTAL PROCEDURE}

\section{Preparation of carbonate crystals}

Aqueous solution containing $10 \mathrm{~mm}$ of PAA $\left(\mathrm{M}_{\mathrm{W}} 250000\right)$ was prepared with purified water. The concentration was calculated based on the monomer unit. After the dissolution of PAA, 5-10 mM of calcium chloride was added to the PAA aqueous solution. The precursor solution containing calcium chloride and PAA $\left(50 \mathrm{~cm}^{3}\right)$ was poured into a polypropylene vessel $\left(60 \mathrm{~cm}^{3}\right)$. Two
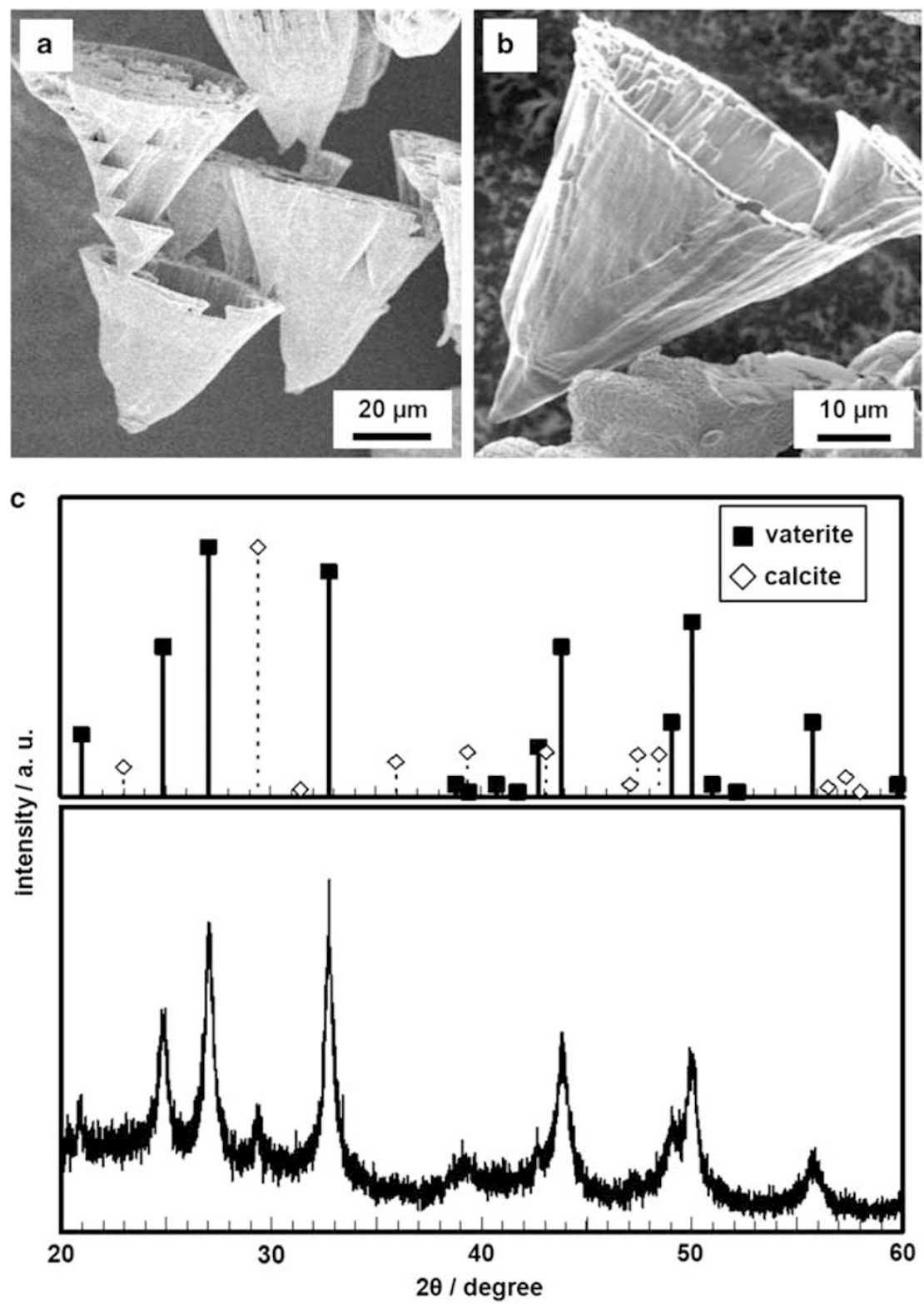

Figure 1 FESEM images ( $\mathbf{a}$ and $\mathbf{b}$ ) and X-ray diffraction (XRD) pattern (c) of the vaterite hollow-cone crystals. 

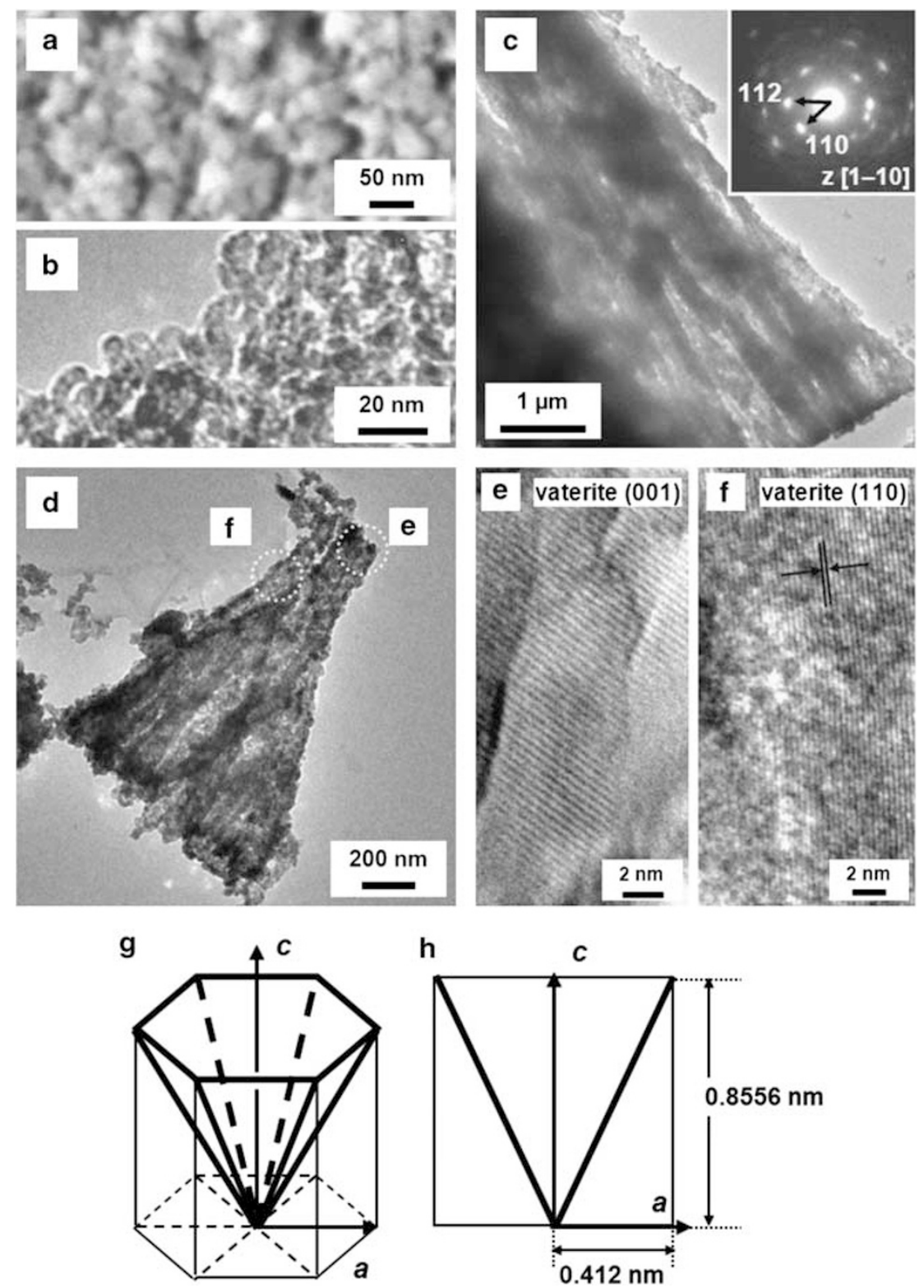

Figure 2 Mesocrystal structures and the crystallographic orientation of the vaterite hollow-cone morphologies. (a and b) Magnified FESEM and fieldemission transmission electron microscopy (FETEM) images, respectively. (c) FETEM image with the SAED pattern (inset). (d-f) FETEM and high-resolution transmission electron microscopy (HRTEM) images. (g) The proposed structural model based on the unit cell of vaterite. (h) The projection of the proposed model in the panel $(\mathbf{g})$ viewed from the (210) direction.

polypropylene vessels were placed in a polystyrene container $\left(700 \mathrm{~cm}^{3}\right)$. Two polypropylene bottles containing $4 \mathrm{~g}$ of ammonium carbonate were sealed with parafilm that was perforated so that five pinholes were created. The two sample bottles of ammonium carbonate were introduced and subsequently placed in the polystyrene container. After sealing, the polystyrene container was maintained at $25^{\circ} \mathrm{C}$ for $3-5$ days. The resulting precipitates at the bottom of the sample vessels were washed by purified water and dried at $25^{\circ} \mathrm{C}$ thereafter. To form calcite crystals, the precursor solution was poured into a glass vessel $\left(60 \mathrm{~cm}^{3}\right)$. To synthesize the aragonite polymorphs, the precursor solution containing $\mathrm{BaCl}_{2} \cdot 6 \mathrm{H}_{2} \mathrm{O}$ in a polypropylene bottle was used instead of the precursor solution containing calcium chloride.

\section{Characterization}

The crystal phases were analyzed by powder X-ray diffraction (Bruker AXS D8Advance, Bruker, Yokohama, Japan). The crystal morphologies were observed by field-emission scanning electron microscopy (FESEM, Hitachi S-4700
(Hitachi, Tokyo, Japan) and FEI Sirion (FEI, Tokyo, Japan) operated at $5.0 \mathrm{kV}$ ). Prior to observation, the FESEM samples were coated with amorphous osmium to ensure their conductivity. The detailed structures were analyzed by field-emission transmission electron microscopy (Tecnai F20, FEI, operated at $200 \mathrm{kV}$ ).

\section{RESULTS AND DISCUSSION}

Hollow-cone morphologies of vaterite $\mathrm{CaCO}_{3}$

Figure 1 shows the resultant hollow-cone $\mathrm{CaCO}_{3}$ crystals. Multiple and single cones were observed in the FESEM images (Figures 1a and $\mathrm{b}$ ). Based on the X-ray diffraction pattern, the polymorphs of the hollow-cone architectures were vaterite, even though a small amount of calcite was observed (Figure 1c). The magnified FESEM and fieldemission transmission electron microscopy images show that these architectures consisted of nanocrystals measuring approximately 

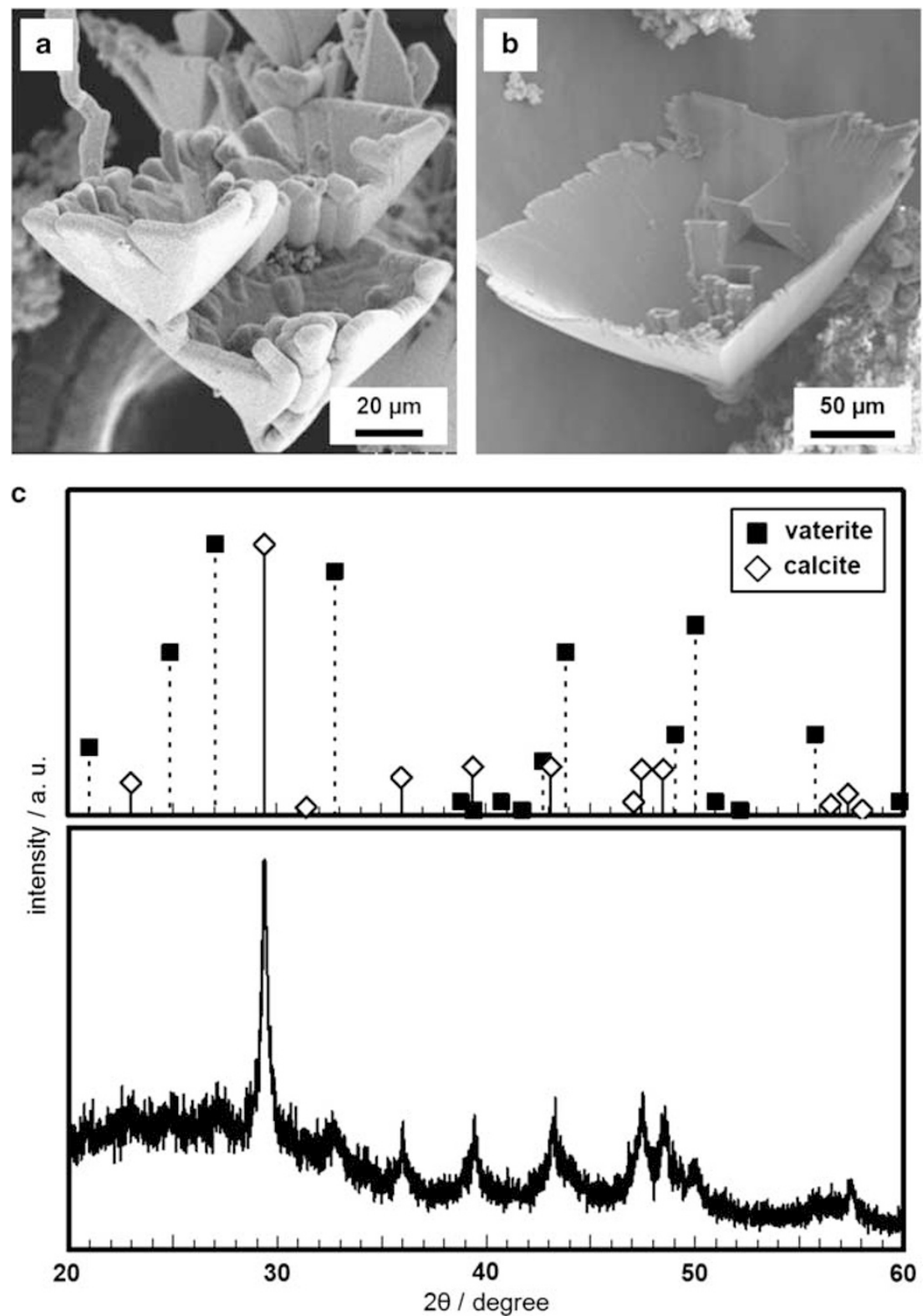

Figure 3 FESEM images ( $\mathbf{a}$ and $\mathbf{b}$ ) and XRD pattern (c) of the hollow four-sided calcite pyramid.

$10 \mathrm{~nm}$ in size (Figures 2a and $\mathrm{b}$ ). The field-emission transmission electron microscopy image with the SAED pattern suggests that the nanocrystals formed the oriented structures, namely, mesocrystals (Figure 2c). The growth direction of the hollow-cone vaterite was analyzed by high-resolution transmission electron microscopy and Fourier transforms (Figures $2 \mathrm{c}-\mathrm{h}$ ). The high-resolution transmission electron microscopy (HRTEM) images show lattice fringes corresponding to the (001) and (110) planes (Figures 2e and f). It is inferred that the cones are grown and opened along the $c$-axis direction as the central axis, as shown in Figures $2 \mathrm{~g}$ and $\mathrm{h}$. Based on the opened angle of the cone, the crystal face exposed on the side face is estimated to correspond to the (101) planes of vaterite (Figures $2 \mathrm{~h}$ ).

Hollow four-sided pyramids of calcite $\mathrm{CaCO}_{3}$

When the sample vessels were moved from polypropylene to glass bottles, four-sided pyramids of calcite with hollow interiors were obtained in the glass bottles (Figure 3). Multiple and single hollow pyramids were formed under the same conditions (Figures $3 a$ and $b$ ). Based on the X-ray diffraction pattern, the main product was calcite, even though a small amount of vaterite was observed (Figure 3c). The magnified FESEM image shows that these architectures consisted of unit crystals $<500 \mathrm{~nm}$ in size (Figure 4a). The magnified fieldemission transmission electron microscopy images show that the unit crystals were formed by the subunit crystals $<$ approximately $10 \mathrm{~nm}$ in size (Figure $4 \mathrm{~b}$ ). These results indicate that the hollow-pyramid shapes were mesocrystals of calcite. In the high-resolution transmission electron microscopy images, the lattice fringes of the (110) planes were observed near the edges (Figures $4 \mathrm{~b}$ and $\mathrm{c}$ ). When the hollow four-sided pyramids were observed from the opened direction, parallelograms were observed along the opening edges (Figure 4d). Based on these results, we developed a model of the hollow four-sided pyramids with the unit cell structure of calcite (Figures $4 \mathrm{e}$ and $\mathrm{f}$ ). The hollow pyramids are grown 

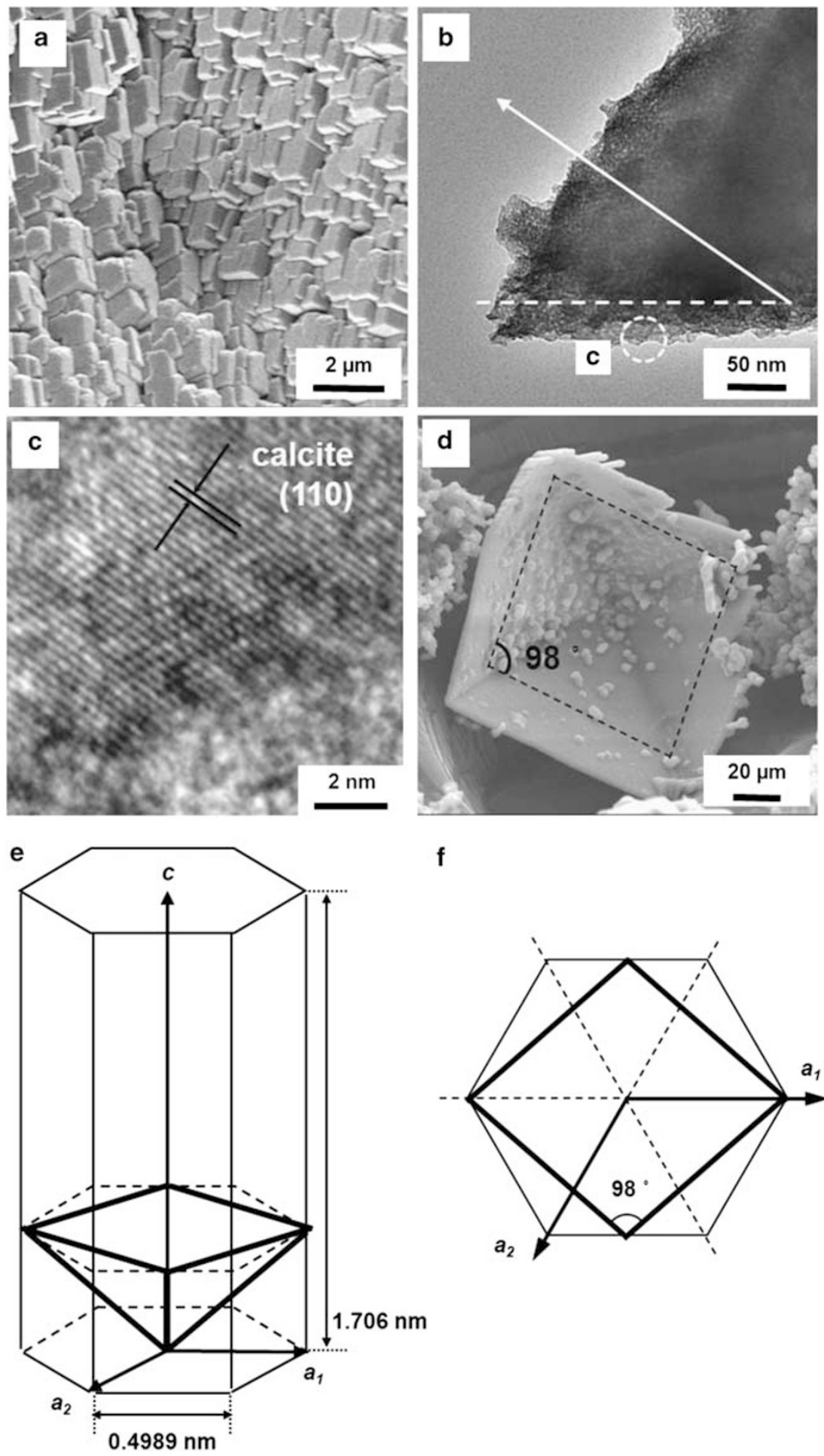

f

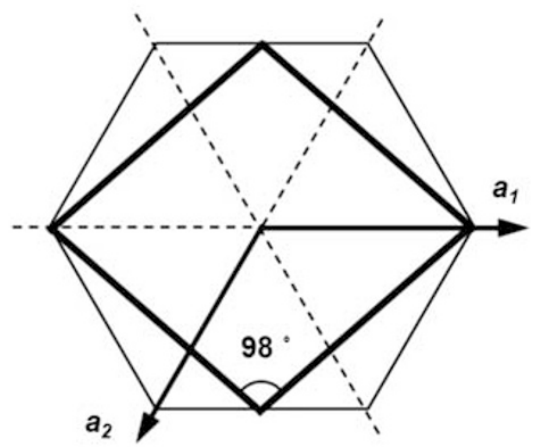

Figure 4 Mesocrystal structures and crystallographic orientation of the hollow four-sided calcite pyramids. (a and b) The magnified FESEM and FETEM images, respectively. (c) HRTEM images. (d) FESEM image viewed from the central axis of the pyramid shape. (e) The proposed structural model based on the unit cell of calcite. (f) The projection viewed from the $c$-axis.

and opened along the $c$-axis direction as the central axis (Figure 4e). The side faces of the cones are ascribed to the (001) plane. The parallelogram of the opening edge viewed from the $c$-axis in the structural model shows the same shape as that observed in the FESEM image (Figures $4 \mathrm{~d}$ and $\mathrm{f}$ ).
Hollow-cone morphologies of aragonite $\mathrm{BaCO}_{3}$

$\mathrm{BaCO}_{3}$ and strontium carbonate $\left(\mathrm{SrCO}_{3}\right)$ have only aragonite polymorphs, regardless of whether the sample vessels used were glass or polypropylene bottles. We used $\mathrm{BaCO}_{3}$ to prepare the hollow-cone shapes consisting of the aragonite polymorph. The alkaline metal 

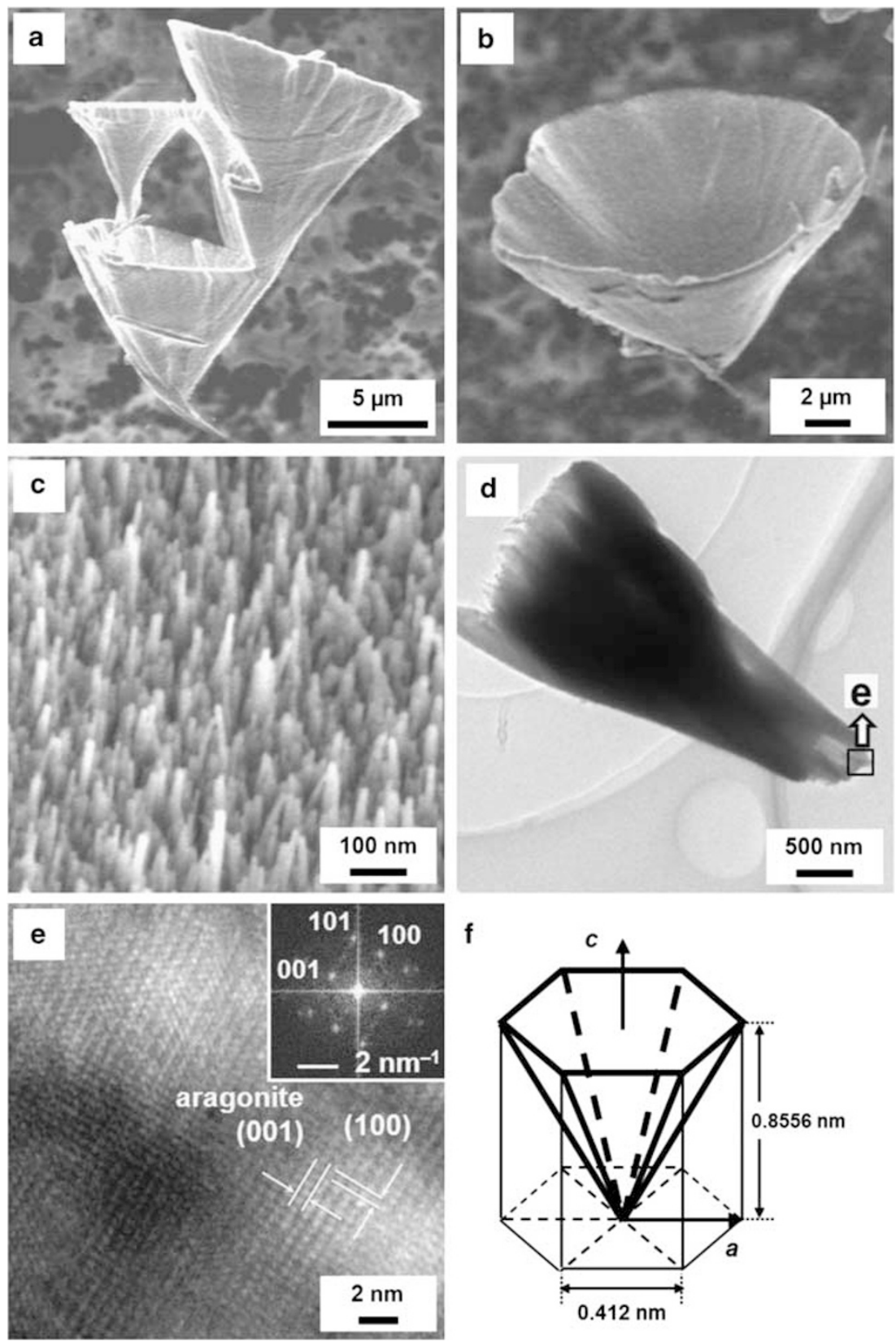

Figure 5 (a-c) FESEM, (d) FETEM and (e) HRTEM images and the proposed structural model (f) of the aragonite $\mathrm{BaCO}_{3}$ hollow-cone shapes.

source was changed from calcium chloride to barium chloride. The other experimental conditions were the same as those used to produce vaterite $\mathrm{CaCO}_{3}$ with a polypropylene sample bottle. The resultant aragonite $\mathrm{BaCO}_{3}$ showed multiple- and single-hollow-cone morphologies (Figures $5 \mathrm{a}$ and $\mathrm{b}$ ). The magnified FESEM images show that the hollow-cone shapes consisted of oriented needle-like aragonite crystals measuring approximately $100 \mathrm{~nm}$ in length and $10 \mathrm{~nm}$ in diameter (Figure 5c). The lattice fringes of the (100) and (001) planes were observed at the starting point of the hollow cones (Figures $5 \mathrm{~d}$ and e). Therefore, the hollow-cone shapes of aragonite $\mathrm{BaCO}_{3}$ are opened along the $c$-axis direction as the central axis (Figure 5f). As well as the vaterite hollow cones, the side faces can be ascribed to the (101) planes of aragonite (Figure 5f).

\section{Proposed formation mechanisms of the hollow architectures}

In the present work, we obtained similar macroscopic hollow-cone architectures consisting of carbonate mesocrystals, regardless of the polymorphs. The results indicate the presence of mutual formation processes involving the molecular-control processes of PAA. At nanoscopic scales, the carbonate crystals form mesocrystal structures with the incorporation of PAA. Thermogravimetry (TG) analysis indicates that approximately $3 \%$ of PAA was incorporated into the hollow-cone architectures of vaterite. It is inferred that the mesocrystal structures were formed from the following processes. ${ }^{17}$ The adsorption of PAA inhibits crystal growth. Because the growth is not completely inhibited by the adsorption of PAA under the supersaturated condition, the growth is restarted by the formation 


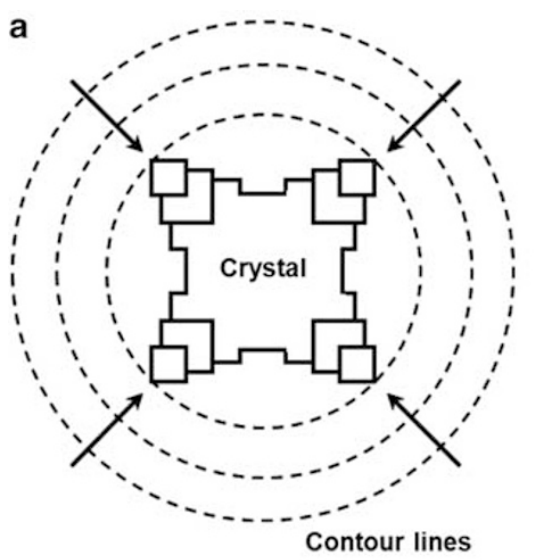

b
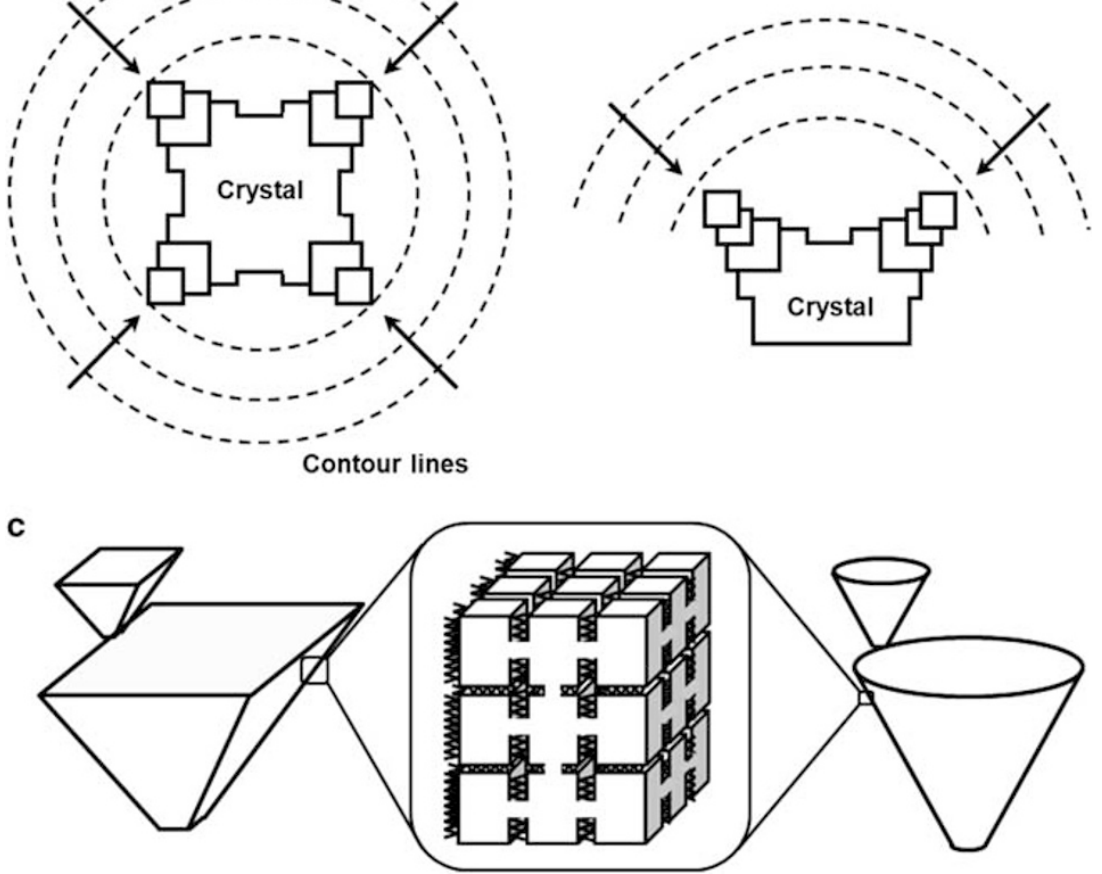

Figure 6 Schematic illustrations of the growth models of the hollow-cone architectures. (a) A two-dimensional growth model of typical skeletal crystals under diffusion-controlled conditions (Berg effect). (b) A two-dimensional growth model of skeletal crystals leading to the hollow architectures in an anisotropic diffusion field. (c) Three-dimensional growth of the skeletal shape leading to the formation of hollow pyramids and conical shapes based on mesocrystals.

of a nanoscale mineral bridge. In this way, segmented growth with periodical inhibition facilitates the formation of the oriented nanocrystals with the incorporation of PAA. ${ }^{9,17}$ Because mesocrystal structures are formed by nanocrystals as the building blocks, a variety of shapes can be directed through the self-organized growth of the nanocrystals. In nature, a variety of macroscopic morphologies are found in biominerals based on carbonate crystals. In artificial systems, mesocrystal structures facilitate the formation of a variety of morphologies. Calcite is obtained when the reaction vessel is changed from polypropylene to glass. Using glass as a substrate or the dissolved silicate species may influence the formation of calcite.

When the precursor solution containing calcium ions and PAA was stirred, the hollow-cone architectures were not obtained. This finding suggests that the diffusion-controlled environment surrounding the crystals has an important role in morphogenesis at macroscopic scales. In the present work, the diffusion-controlled condition was achieved by several factors as follows. A carbonate source was introduced through the decomposition of ammonium carbonate outside of the sample vessel. The presence of PAA limits the diffusion of calcium ions because of the complexation or the viscosity of the solution. Therefore, the supply of calcium and carbonate ions may lead to the diffusion-controlled condition.

In general, the diffusion-controlled conditions required for crystal growth are achieved in a polymer matrix. Kato and co-workers reported that patterned structures were created upon the crystallization of $\mathrm{CaCO}_{3}$ in polymer matrices. ${ }^{26,27}$ In our previous reports, ${ }^{9,32}$ the morphologies of crystals were transformed from those of polyhedral shapes to dendritic morphologies via skeletal forms as the density of the polymer matrices increased. The diffusioncontrolled conditions generate a concentration gradient around the polyhedral shapes. Therefore, the growth of the edges was promoted to induce the formation of the skeletal morphologies under the diffusion-controlled conditions (Berg effect, Figure 6a). ${ }^{9}$ The formation mechanism of the hollow-cone architecture is similar to that of the skeletal structures under the diffusion-controlled conditions (Figure 6a). Typical skeletal crystals are isotropically grown along the concentration gradient around the polyhedral shapes based on typical crystal behavior (Figure 6a). If the diffusion direction is limited around the polyhedral shape, the growth direction of the skeletal morphology is also limited along anisotropic directions (Figure 6b). In the present work, the hollow architectures were obtained as precipitates at the bottom of the sample vessels. This result implies that the diffusion direction is limited. The anisotropic diffusion of ions directs the morphogenesis of the macroscopic conical shapes through the formation of the mesocrystals. Thus, hollow four-sided pyramids exhibiting typical calcite-crystal behavior were formed (Figure 6c). Because the nanocrystals in the mesocrystal structure serve as building blocks, the morphogenesis of the macroscopic shapes is not limited to the shapes originating from the typical crystal behavior. Therefore, the hollow-cone shapes of vaterite and aragonite were obtained under diffusion-controlled conditions.

\section{SUMMARY}

The hollow-cone architectures of carbonate crystals, such as calcite and vaterite $\mathrm{CaCO}_{3}$ and aragonite $\mathrm{BaCO}_{3}$, were synthesized through a molecular-control approach in the presence of an organic polymer. Oriented nanocrystals made up the macroscopic hollow-cone shapes, regardless of the polymorphs of the carbonate crystals. Regarding the molecular control in the present work, PAA has important roles in the formation of the hierarchically organized architectures. The mesocrystal structures are formed through the mediation of PAA on the 
faces of the nanocrystals. On macroscopic scales, diffusion-controlled conditions are achieved to induce the preferential growth of the edges, similar to the morphogenesis of skeletal crystals. Therefore, the hierarchically organized and hollow-cone architectures can be synthesized through a molecular-control approach. This synthetic strategy can be applied to other combinations of crystals and polymers. Furthermore, the mechanisms underlying this phenomenon may be relevant to the morphogenesis of biominerals.

\section{ACKNOWLEDGEMENTS}

This work was partially supported by a Grant-in-Aid for Scientific Research (no. 22107010) on the Innovative Areas: 'Fusion Materials' (area no. 2206) from the Ministry of Education, Culture, Sports, Science and Technology (MEXT).

1 Mann, S. Molecular recognition in biomineralization. Nature 332, 119-124 (1988).

2 Addadi, L. \& Weiner, S. Control and design principles in biological mineralization. Angew. Chem. Int. Ed. Engl. 31, 153-169 (1992).

3 Bäuerlein, E., Behrens, P. \& Epple, M. Handbook of Biomineralization (Wiley-VCH, Weinheim (2007).

4 Mann, S. Molecular tectonics in biomineralization and biomimetic materials chemistry. Nature 365, 499-505 (1993).

5 Kato, T. Polymer/calcium carbonate layered thin-film composites. Adv. Mater. 12 1543-1546 (2000).

6 Naka, K. \& Chujo, Y. Control of crystal nucleation and growth of calcium carbonate by synthetic substrates. Chem. Mater. 13, 3245-3259 (2001).

7 Kato, T., Sugawara, A. \& Hosoda, N. Calcium carbonate-organic hybrid materials. Adv. Mater. 14, 869-877 (2002).

8 Yu, S.-H. \& Cölfen, H. Bio-inspired crystal morphogenesis by hydrophilic polymers. J. Mater. Chem. 14, 2124-2147 (2004).

9 Imai, H., Oaki, Y. \& Kotachi, A. A biomimetic approach for hierarchically structured inorganic crystals through self-organization with polymeric molecules. Bull. Chem. Soc. Jpn. 79, 1834-1851 (2006).

10 Xu, A. W., Ma, Y. \& Cölfen, H. Biomimetic mineralization. J. Mater. Chem. 17 415-449 (2007).

11 Meldrum, F. C. \& Cölfen, H. Controlling mineral morphologies and structures in biological and synthetic systems. Chem. Rev. 108, 4332-4432 (2008).

12 Sommerdijk, N. A. J. M. \& de With, G. Biomimetic $\mathrm{CaCO}_{3}$ mineralization using designer molecules and interfaces. Chem. Rev. 108, 4499-4550 (2008).

13 Kato, T., Sakamoto, T. \& Nishimura, T. Macromolecular templating for the formation of inorganic-organic hybrid structures. MRS Bull. 35, 127-132 (2010).

14 Imai, H. \& Oaki, Y. Bioinspired hierarchical crystals. MRS Bull. 35, 138-144 (2010).

15 Oaki, Y. \& Imai, H. The hierarchical architecture of nacre and its mimetic material. Angew. Chem. Int. Ed. 44, 6571-6575 (2005).

16 Oaki, Y. \& Imai, H. Nanoengineering in echinoderms: emergence of morphology from nanobricks. Small 2, 66-71 (2006).

17 Oaki, Y., Kotachi, A., Miura, T. \& Imai, H. Bridged nanocrystals in biominerals and their biomimetics: classical yet modern crystal growth on the nanoscale. Adv. Funct. Mater. 16, 1633-1639 (2006).

18 Cölfen, H. \& Antonietti, M. Mesocrystals: Inorganic superstructures made by highly parallel crystallization and controlled alignment. Angew. Chem. Int. Ed. 44, 5576-5591 (2005)
19 Niederberger, M. \& Cölfen, H. Oriented attachment and mesocrystals: non-classical crystallization mechanisms based on nanoparticle assembly. Phys. Chem. Phys. Chem. 8, 3271-3287 (2006).

20 Zhou, L. \& O'Brien, P. Mesocrystals: a new class of solid materials. Small 4, 1566-1574 (2008).

21 Kato, T., Suzuki, T., Amamiya, T., Irie, T. \& Komiyama, M. Effects of macromolecules on the crystallization of $\mathrm{CaCO}_{3}$ : the formation of organic/inorganic composites. Supramol. Sci. 5, 411-415 (1998).

22 Hosoda, N., Sugawara, A. \& Kato, T. Template effect of crystalline poly(vinyl alcohol) for selective formation of aragonite and vaterite $\mathrm{CaCO}_{3}$ thin films. Macromolecules 36, 6449-6452 (2003).

23 Sugawara, A., Ishii, T. \& Kato, T. Self-organized calcium carbonate with regular surface relief structures. Angew. Chem. Int. Ed. 42, 5299-5303 (2003).

24 Sugawara, A., Nishimura, T., Yamamoto, Y., Inoue, H., Nagasawa, H. \& Kato, T. Selforganization of oriented calcium carbonate/polymer composites: effects of a matrix peptide isolated from the exoskeleton of a crayfish. Angew. Chem. Int. Ed. 45, 2876-2879 (2006).

25 Nishimura, T., Ito, T., Yamamoto, Y., Yoshio, M. \& Kato, T. Macroscopically ordered polymer $/ \mathrm{CaCO}_{3}$ hybrids prepared by using a liquid-crystalline template. Angew. Chem. Int. Ed. 47, 2800-2803 (2008).

26 Sakamoto, T., Oichi, A., Nishimura, T., Sugawara, A. \& Kato, T. Calcium carbonate/ polymer thin-film hybrids: induction of the formation of patterned aragonite crystals by thermal treatment of a polymer matrix. Polym. J. 41, 522-523 (2009).

27 Sakamoto, T., Oichi, A., Oaki, Y. Nishimura, T., Sugawara, A \& Kato, T. Threedimensional relief structures of $\mathrm{CaCO}_{3}$ crystal assemblies formed by spontaneous twostep crystal growth on a polymer thin film. Cryst. Growth Des. 9, 622-625 (2009).

28 Yamamoto, Y., Nishimura, T., Saito, T. \& Kato, T. $\mathrm{CaCO}_{3} /$ chitin-whisker hybrids: formation of $\mathrm{CaCO}_{3}$ crystals in chitin-based liquid-crystalline suspension. Polym. J. 42, 583-586 (2010)

29 Kotachi, A. \& Imai, H. Polymorph control of calcium carbonate films in a poly(acrylic acid)-chitosan system. Cryst. Growth Des. 6, 1636-1641 (2006).

30 Takaguchi, Y., Yanagimoto, Y., Tajima, T., Ohta, K., Motoyoshiya, J. \& Aoyama, H. Photodimerization of anthracene having dendritic substituent within the vaterite crystals of $\mathrm{CaCO}_{3}$. Chem. Lett. 1102-1103 (2002).

31 Oaki, Y. \& Imai, H. Hierarchically organized superstructure emerging from exquisite association of inorganic crystals, organic polymers, and dyes: a model approach toward suprabiomineral material. Adv. Funct. Mater. 15, 1407-1414 (2005).

32 Oaki, Y. \& Imai, H. Experimental demonstration for the morphological evolution of crystals grown in gel media. Cryst. Growth Des. 3, 711-716 (2003).

33 Oaki, Y., Hayashi, S. \& Imai, H. A hierarchical self-similar structure of oriented calcite with association of an agar gel matrix: inheritance of crystal habit from nanoscale. Chem. Commun. 2841-2843 (2007).

34 Qi, L., Cölfen, H., Antonietti, M., Li, M., Hopwood, J. D., Ashley, A. J. \& Mann, S. Formation of $\mathrm{BaSO}_{4}$ fibres with morphological complexity in aqueous polymer solutions. Chem. Eur. J. 7, 3526-3532 (2001).

$35 \mathrm{Yu}, \mathrm{S}$. H., Antonietti, M., Cölfen, H. \& Hartmann, J. Growth and self-assembly of $\mathrm{BaCrO}_{4}$ and $\mathrm{BaSO}_{4}$ nanofibers toward hierarchical and repetitive superstructures by polymer-controlled mineralization reactions. Nano. Lett. 3, 379-382 (2003).

36 Rudloff, J. \& Cölfen, H. Superstructures of temporarily stabilized nanocrystalline $\mathrm{CaCO}_{3}$ particles: Morphological control via water surface tension variation. Langmuir 20, 991-996 (2004).

37 Fan, Y. \& Wang, R. Submicrometer-sized vaterite tubes formed through nanobubbletemplated crystal growth. Adv. Mater. 17, 2384-2388 (2005).

38 Mukkamala, S. B. \& Powell, A. K. Biomimetic assembly of calcite microtrumpets: crystal tectonics in action. Chem. Commun. 918-919 (2004).

$39 \mathrm{Wu}$, C., Yu, S. H. \& Antonietti, M. Complex concaved cuboctahedrons of copper sulfide crystals with high geometrical symmetry created by a solution process. Chem. Mater. 18, 3599-3601 (2006).

40 Zhu, J. P., Yu, S. H., He, Z. B., Jiang, J., Chen, K. \& Zhou, X. Y. Complex PbTe hopper (skeletal) crystals with high hierarchy. Chem. Commun. 5802-5804 (2005). 